\title{
Heart rate variability measurement and clinical depression in acute coronary syndrome patients: narrative review of recent literature
}

This article was published in the following Dove Press journal:

Neuropsychiatric Disease and Treatment

18 July 2014

Number of times this article has been viewed

\author{
Patricia RE Harris' \\ Claire E Sommargren ${ }^{2}$ \\ Phyllis K Stein ${ }^{3}$ \\ Gordon L Fung ${ }^{4,5}$ \\ Barbara J Drew 6,7 \\ 'ECG Monitoring Research Lab, \\ ${ }^{2}$ Department of Physiological Nursing, \\ School of Nursing, University of \\ California, San Francisco, CA, USA; \\ ${ }^{3}$ Heart Rate Variability Laboratory, \\ School of Medicine, Washington \\ University, St Louis, MO, USA; \\ ${ }^{4}$ Asian Heart \& Vascular Center at \\ Mount Zion, Division of Cardiology, \\ University of California, ${ }^{5}$ Cardiology \\ Consultation Service, Cardiac \\ Noninvasive Laboratory, and The \\ Enhanced External Counterpulsation \\ Unit, Department of Medicine, \\ University of California, San \\ Francisco Medical Center, ${ }^{6}$ Division \\ of Cardiology, ${ }^{7}$ Department of \\ Physiological Nursing, School of \\ Nursing, University of California, San \\ Francisco, CA, USA
}

Correspondence: Patricia RE Harris University of California, San Francisco, 2128 Blue Jay Circle, Pinole,

CA 94564, USA

Tel +I 5102607307

Email patriciarae.harris@ucsf.edu
Aim: We aimed to explore links between heart rate variability (HRV) and clinical depression in patients with acute coronary syndrome (ACS), through a review of recent clinical research literature.

Background: Patients with ACS are at risk for both cardiac autonomic dysfunction and clinical depression. Both conditions can negatively impact the ability to recover from an acute physiological insult, such as unstable angina or myocardial infarction, increasing the risk for adverse cardiovascular outcomes. HRV is recognized as a reflection of autonomic function.

Methods: A narrative review was undertaken to evaluate state-of-the-art clinical research, using the PubMed database, January 2013. The search terms "heart rate variability" and "depression" were used in conjunction with "acute coronary syndrome", "unstable angina", or "myocardial infarction" to find clinical studies published within the past 10 years related to HRV and clinical depression, in patients with an ACS episode. Studies were included if HRV measurement and depression screening were undertaken during an ACS hospitalization or within 2 months of hospital discharge.

Results: Nine clinical studies met the inclusion criteria. The studies' results indicate that there may be a relationship between abnormal HRV and clinical depression when assessed early after an ACS event, offering the possibility that these risk factors play a modest role in patient outcomes.

Conclusion: While a definitive conclusion about the relevance of HRV and clinical depression measurement in ACS patients would be premature, the literature suggests that these measures may provide additional information in risk assessment. Potential avenues for further research are proposed.

Keywords: autonomic nervous system, depressive disorder, outcomes research, risk assessment

\section{Introduction}

Relieving the burden of morbidity and mortality in cardiovascular (CV) disease is a vital, ongoing public health goal. While great progress has been made in managing acute coronary syndrome (ACS), the incidence of coronary events remains high. In 2010, over 1.1 million hospitalizations in the United States were attributed to ACS, the umbrella term for myocardial infarction (MI) or unstable angina (UA). ${ }^{1}$ Recognition and management of $\mathrm{CV}$ risk factors promotes tertiary prevention, potentially helping patients avoid repeated ACS episodes.

Autonomic nervous system dysfunction ${ }^{2-6}$ and clinical depression ${ }^{7-10}$ have been associated with a significant risk of adverse CV outcomes in ACS patients. Cardiac autonomic physiology, as measured by heart rate variability (HRV), appears to 
have a complex interplay with psychosocial factors. ${ }^{2,11,12}$ Understanding the association of altered HRV and depression, as a possible means to improve the prognosis of ACS patients, has become the focus of recent studies. ${ }^{13-21}$

\section{Background}

Research has linked the human autonomic response to threat - increased sympathetic and decreased parasympathetic activity - with a higher risk of sudden cardiac death in the presence of MI. ${ }^{3,4,22}$ UA patients also may have an increased risk for adverse $\mathrm{CV}$ events. ${ }^{5} \mathrm{HRV}$ serves as a quantitative reflection of autonomic nervous system activity and has been shown to have potential for risk stratification in cardiac patients. ${ }^{2,23-25}$

Clinical depression also has been linked to adverse CV outcomes, including death. ${ }^{7-9}$ Of patients hospitalized for acute $\mathrm{MI},>30 \%$ are reported to have mild-to-moderate depressive symptoms, and nearly $20 \%$ have major depression. ${ }^{10}$ The mechanisms behind the connection between depression and post-MI mortality are not yet clear. ${ }^{26}$ Study results describing the full extent of the role of depression in adverse outcomes have been inconsistent. ${ }^{27}$

In 1988, Carney et al examined depression and HRV in stable coronary artery disease and found that HRV was lower in the patients with coronary artery disease who were depressed than in those who were nondepressed. ${ }^{28}$ In the ensuing years, further research has suggested an adverse association between HRV and depression in patients with ACS. ${ }^{11,13-15,17-21,29}$

\section{The review}

\section{Aims}

The primary aim of this review was to examine current research findings regarding links between HRV and clinical depression in ACS patients, assessed during or soon after hospital admission. The reasons for clinical depression are multifaceted, and the underlying physiology is not well understood. ${ }^{26,27}$ Screening for depression in ACS patients is recommended in practice guidelines. ${ }^{30,31}$ The research literature raises a question: Could the assessment of HRV in conjunction with depression screening, potentially during ACS hospitalization or soon afterward, offer information to assist in assessing risk? The secondary aim of this review was to increase knowledge about HRV and its measurement. To address the second aim, the history of HRV in relation to ACS, and HRV measurement are discussed in the sections preceding the "Results" section. Table 1 provides the definitions of the HRV variables.

\section{Design}

While several reviews have examined depression and HRV in relation to stable coronary heart disease, their early assessment in ACS has received little attention. The uniqueness of our narrative review lies in its focus on current studies that measured HRV and clinical depression during or within 2 months of ACS hospitalization, the time frame typically required for ACS patients to move beyond the highly acute phase. ${ }^{31}$ Our pragmatic rationale was that cardiac patients are monitored routinely from the time they enter the hospital, and electrocardiographic (ECG) monitoring is recommended for at least 24 hours. ${ }^{32} \mathrm{HRV}$ measurements generally can be computed from a continuous ECG record. ${ }^{24}$ In addition to assessing HRV, screening ACS patients for clinical depression during their hospitalization could be a practical approach to enhancing outcomes after discharge.

\section{Search methods}

We searched the PubMed and Web of Science databases to identify key clinical studies related to HRV measurement and depression, in patients with a recent ACS episode. The search terms "heart rate variability" and "depression" were used in conjunction with "acute coronary syndrome", "unstable angina", or "myocardial infarction". The limiters "human", "adult", and "English" were used. A publication time frame within the preceding 10 years was considered reasonable to help ensure state-of-the-art findings.

\section{Search outcomes}

After duplicates from the different searches were eliminated, 12 unique data-based clinical articles related to HRV and depression in ACS were examined. Three studies in which initial HRV or depression was measured outside the 2-month time frame were excluded. Five articles that reported initial in-hospital measurements were retained for review, along with four studies that described initial HRV and depression assessments performed within 2 months of discharge. The nine studies that met the inclusion criteria were observational or secondary analyses.

\section{Quality appraisal}

All the articles in the review focused upon the research question. The search and selection methods, inclusion/ exclusion criteria, selection of articles, assessment of the quality of the literature, data synthesis, construction of a representational schematic (Figure 1), and conclusions were assessed and approved by the authors of this review. 
Table I Definitions of frequently used heart rate variability (HRV) measurements

\begin{tabular}{|c|c|}
\hline Variable abbreviation & HRV definitions (unit of measurement) \\
\hline \multicolumn{2}{|c|}{ Time domain variables: measured using descriptive statistics ${ }^{5,27}$} \\
\hline \multicolumn{2}{|c|}{ Long-term time domain measures } \\
\hline SDNN & $\begin{array}{l}\text { Standard deviation }(\mathrm{SD}) \text { of all normal RR intervals (Normal-Normal }[\mathrm{NN}] \text { ) usually measured over } \sim 24 \\
\text { hours (milliseconds [ms]) (estimates global heart rate variability power; corresponds to total power) }\end{array}$ \\
\hline SDANN & $\begin{array}{l}\text { Standard deviation of the average normal RR (NN) intervals in all 5-minute segments, usually measured } \\
\text { over } \sim 24 \text { hours (ms) (corresponds to ULF) }\end{array}$ \\
\hline SDNN index & $\begin{array}{l}\text { Mean of the standard deviations of normal RR (NN) intervals in all 5-minute segments } \\
\text { over } \sim 24 \text { hours (ms) (corresponds to the mean of 5-minute segments of total power) }\end{array}$ \\
\hline \multicolumn{2}{|c|}{ Short-term time domain measures } \\
\hline rMSSD (rMSNN) & $\begin{array}{l}\text { Square root of the mean of squares of the differences between successive normal RR (NN) intervals } \\
\text { (ms); referred to as rMSNN in the study by Guinjoan et al }{ }^{13} \text { (corresponds to HF) }\end{array}$ \\
\hline pNN50* & $\begin{array}{l}\text { Percentage of normal RR intervals that are }>50 \text { ms different from previous normal RR during } \\
\text { recording time (\%) (corresponds to HF) }\end{array}$ \\
\hline \multicolumn{2}{|c|}{ Frequency domain variables: measured using spectral analysis ${ }^{5,27}$} \\
\hline TP* & Total power, estimates global power, ie, HF, LF, VLF, and ULF, measured over $\sim 24$ hours $\left(\mathrm{ms}^{2}\right.$ ) \\
\hline $\mathrm{HF}^{*}$ & $\begin{array}{l}\text { Average of } 5 \text {-min segments of high frequency power for total recording time }\left(\mathrm{ms}^{2}\right) \text {; HF reference } \\
\text { range }=0.15-0.4 \mathrm{~Hz}\end{array}$ \\
\hline LF* & $\begin{array}{l}\text { Average of } 5 \text {-min segments of low frequency power for the total recording time }\left(\mathrm{ms}^{2}\right) \text {; LF reference } \\
\text { range }=0.04-0.15 \mathrm{~Hz}\end{array}$ \\
\hline \multirow[t]{2}{*}{ VLF* } & Very low frequency power, averages of 5-minute segments measured over the total recording time $\left(\mathrm{ms}^{2}\right)$; \\
\hline & VLF reference range $=0.003-0.04 \mathrm{~Hz}$ \\
\hline ULF* & Ultra low frequency power measured over $\sim 24$ hours $\left(\mathrm{ms}^{2}\right)$; ULF reference range $\leq 0.003 \mathrm{~Hz}$ \\
\hline $\mathrm{LF} / \mathrm{HF}^{*}$ & $\begin{array}{l}\text { Average of } 5 \text {-min segments of normalized low/high frequency ratio: } \\
(\mathrm{LF} /[\text { total power }-\mathrm{VLF}] \times 100) \text { divided by }(\mathrm{HF} /[\text { total power }-\mathrm{VLF}] \times 100)\end{array}$ \\
\hline \multicolumn{2}{|c|}{ Nonlinear measures $9,29,33-36$} \\
\hline ApEn & $\begin{array}{l}\text { Approximate entropy: measure of randomness or disorder within a system; calculation of the } \\
\text { logarithmic likelihood that patterns that are similar remain similar in the next incremental comparisons }\end{array}$ \\
\hline DFAI & $\begin{array}{l}\text { Detrended fractal analysis: short-term fractal scaling exponent calculated over 3-II beats, averaged } \\
\text { over I,000 beats for } 24 \text { hours (also known as alpha-I, reported as an exponent value) }\end{array}$ \\
\hline SDI2 & SDI2, SDI, and SD2 are derived from the Poincare plot (a scatter plot in which each R-R interval is \\
\hline \multirow[t]{3}{*}{ SDI, SD2 } & plotted against the next $\mathrm{R}-\mathrm{R}$ interval). SDI2 is the ratio of the dispersion, or standard deviation, of the \\
\hline & $\begin{array}{l}\text { plot's perpendicular axis, indicating short-term, instantaneous beat-to-beat variance (known as SDI), } \\
\text { versus the standard deviation of the plot's diagonal axis, indicating long-term, continuous variance } \\
\text { (known as SD2) }\end{array}$ \\
\hline & $\begin{array}{l}\text { Note: While the SDI/SD2 ratio is a nonlinear measurement, SDI and SD2, calculated individually, are } \\
\text { linear measurements; SDI correlates precisely with rMSSD }\end{array}$ \\
\hline
\end{tabular}

Notes: The terms "variables," "measures," "measurements," and "indices" are often used interchangeably in referring to HRV. *Natural log transformations are often performed for these variables, which usually are not normally distributed. Time domain HRV measurements are based upon the intervals between the "R" waves in the QRS complexes (referred to as the RR interval). The QRS complex in the ECG corresponds to depolarization of the ventricles.

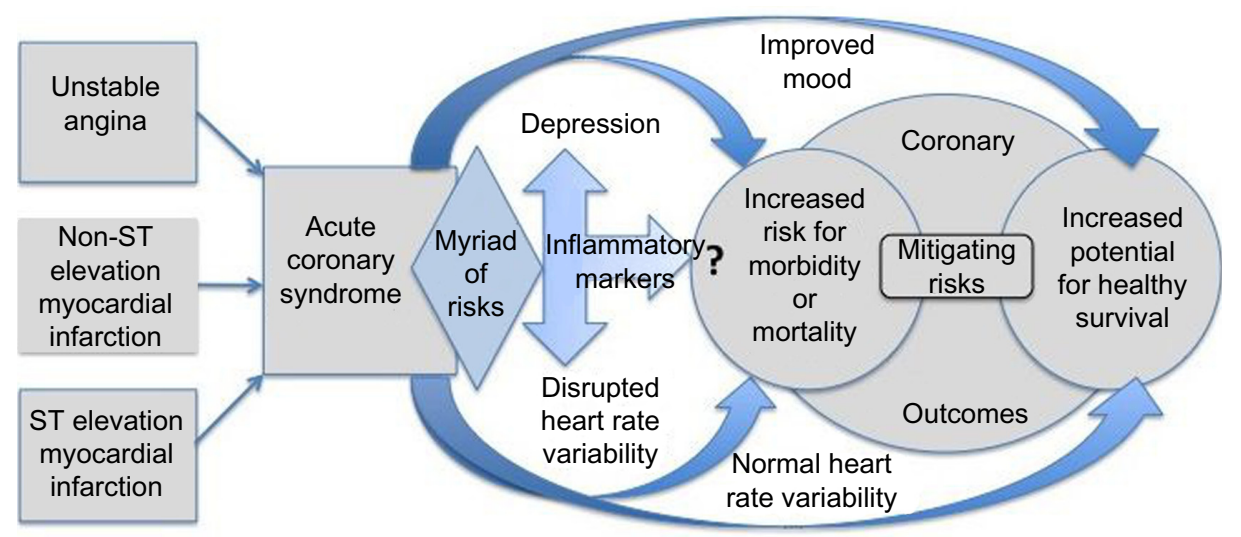

Figure I Representation of potential coronary outcome pathways related to mood and heart rate variability after an episode of acute coronary syndrome, ie, unstable angina, non-ST elevation myocardial infarction, or ST elevation myocardial infarction. 


\section{Data abstraction}

The studies were examined closely. Focus was placed on the methods sections to discern measurement time frames. Table 2 was created to summarize the methods and key findings.

\section{Synthesis}

The authors of this review discussed the possible relationships between ACS patients' depression, autonomic dysfunction (reflected by HRV), and coronary outcomes. The clinical research studies were individually scrutinized and grouped into categories. A schematic offering a visual representation of the relationships between variables was constructed (Figure 1).

\section{History of heart rate variability in relation to acute coronary syndrome}

HRV is based upon the concept of healthy variations in the time intervals between sinus beats on the ECG. ${ }^{2,24,25} \mathrm{~A}$ healthy human heart with an intact autonomic nervous system continuously adjusts to internal and external changes; thus a heart rate of 60 beats per minute does not mean that the heart beats precisely one time per second. Each interval varies slightly by milliseconds. ${ }^{33}$

Researchers have suggested that a decrease in variation between sinus beats may be linked to poor CV outcomes. The potential clinical significance of HRV in predicting outcomes of acute MI was described nearly 50 years ago. In 1965, Schneider and Costiloe presented evidence that MI patients had less sinus arrhythmia - a component of HRV associated with respiration - than did healthy controls. ${ }^{34}$ In 1978, Wolf et al reported that among patients admitted to a coronary care unit (CCU), those showing evidence of sinus arrhythmia upon admission tended to have less mortality and better prognosis. ${ }^{35}$ A seminal 1987 study by Kleiger et al demonstrated that low HRV, measured with Holter recorders (ambulatory electrocardiography device) 2 weeks post-MI, was independently associated with all-cause mortality during 31 months of follow-up. ${ }^{3}$ Since these early findings, research supporting the prognostic value of HRV measured after MI has expanded ${ }^{4,6}$ and includes UA patients, ${ }^{5}$ albeit a causal link between altered HRV and poor ACS outcomes has not been established. ${ }^{2}$

\section{Heart rate variability measurement}

$\mathrm{HRV}$ is a noninvasive measurement that can be obtained from ECG monitoring, commonly 24-hour Holter recordings. 2,24,25 Derived from mathematical formulas and computed with the aid of computer software, HRV has been used primarily as a research tool to assess autonomic function in physiological studies or to examine the cardiac autonomic role in risk stratification. One limitation of HRV assessment is that sinus rhythm is required to make accurate measurements, so patients who have atrial fibrillation or a pacemaker are precluded from analysis. ${ }^{24}$ Despite this constraint, the clinical benefits of HRV analysis have become more widely recognized in recent years. The American College of Cardiology and American Heart Association recommend education and skill in HRV assessment as a prerequisite to competence in ambulatory ECG interpretation. ${ }^{36}$ The American College of Cardiology/American Heart Association 2004 practice guidelines recommend considering the use of HRV to assess the risk of ventricular arrhythmias in the management of patients recovering from ST-elevation MI (class IIb, usefulness is less well established). ${ }^{30}$

HRV measurement methods typically fall into three categories: time domain; frequency domain; and nonlinear dynamics. ${ }^{2,6,24,25}$ HRV definitions are presented in Table 1.

Time domain HRV uses descriptive statistics to estimate the variation in normal sinus RR intervals over time.,24,25 For example, the standard deviation of all normal RR intervals over the length of a recording period is called SDNN. There are two time domain categories: long-term, usually measured over 24 hours, potentially reflecting long cycles, such as circadian rhythm; and short-term, which can be measured over 5-10 minutes, potentially reflecting short cycles of beat-tobeat patterns, such as respiratory or baroreceptor activity. ${ }^{24}$

Frequency domain measures use spectral density analysis to estimate HRV. ${ }^{37}$ Frequency domain HRV is derived from mathematical algorithms and examines the amount of underlying cyclic variation in intervals across time (known as variance or power). ${ }^{24,37}$ The spectrum is divided into four bands: high frequency (HF); low frequency (LF); very low frequency (VLF); and ultra-low frequency (ULF). ${ }^{2,24,25} \mathrm{HF}$ usually synchronizes with the respiratory cycle. ${ }^{38} \mathrm{LF}$ has been associated with changes in blood pressure along with fluctuations in sympathetic and parasympathetic activity. VLF has been related to alterations in body temperature and sleep apnea-related heart rate changes. ${ }^{39}$ ULF has been associated with circadian rhythms. ${ }^{40}$

The third category, nonlinear dynamic measures, takes into account the intricate interactions of physiological signals. ${ }^{41}$ They are computed based upon newer bioengineering developments, incorporating theories of complexity, chaos, and fractal characteristics. ${ }^{39-44}$ These 


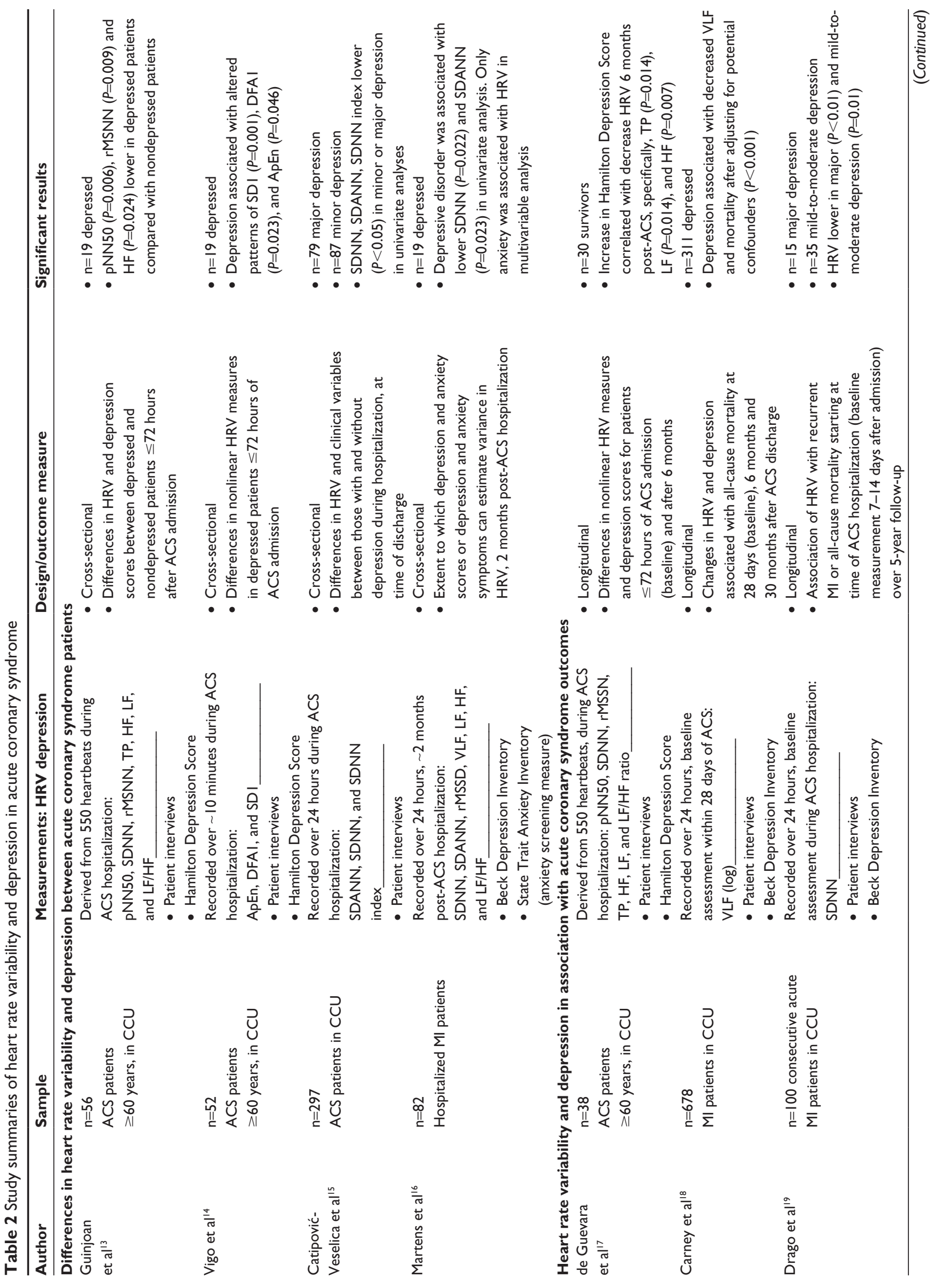




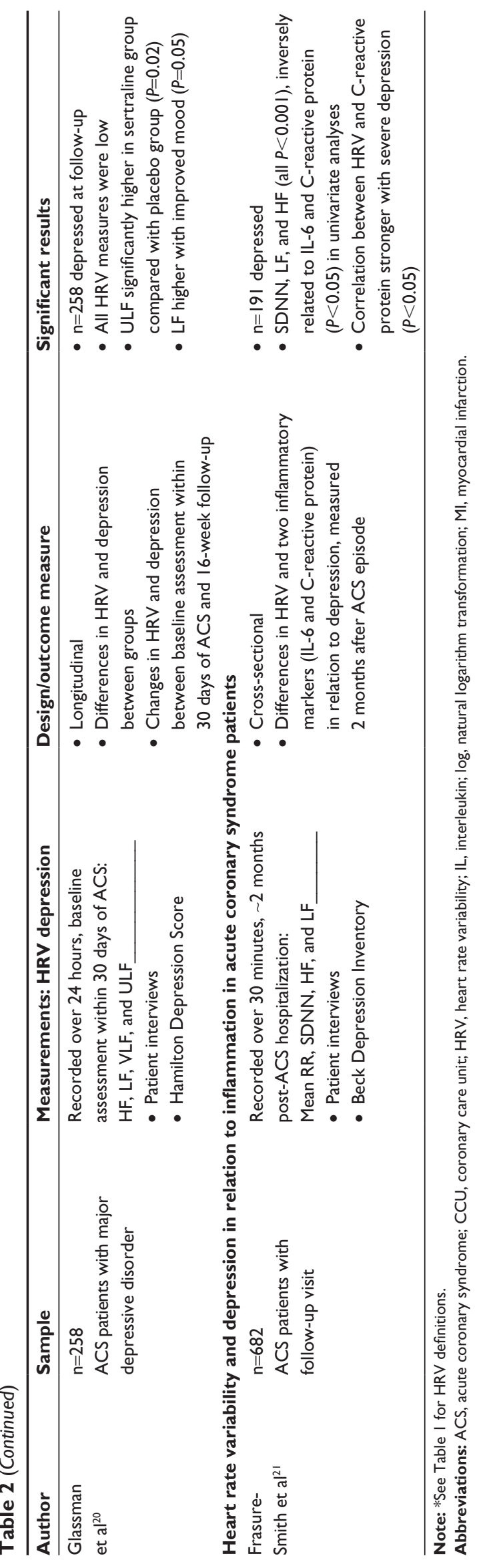

methods may capture information about underlying patterns of physiological variability (such as how random or correlated the signals are) beyond that which time and frequency domain measurements can offer., ${ }^{2,44}$

Overall, HRV variables appear to reflect the interplay between the parasympathetic and sympathetic autonomic branches. Associating specific HRV measures solely with one or the other autonomic branch is inaccurate. ${ }^{2}$ For example, HF power often is ascribed to respiratory sinus arrhythmia, reflecting vagal involvement. While physiological study supports this relationship, ${ }^{38}$ clinical research indicates that this is not true in all patients. Extremely high HF values may not necessarily reflect better parasympathetic control of heart rate but rather, might be related to disrupted heart rate patterns as a consequence of high sympathetic activation or other abnormal, yet to be identified, phenomena. ${ }^{45}$

The studies in this review used standard time and frequency domain measures ${ }^{24}$ or nonlinear dynamic measures to assess HRV. These measures have been supported by previous clinical research ${ }^{43,44}$ and may have potential for clinical applications..$^{46,47}$

\section{Results}

The studies were organized into three groups, based upon HRV and depression measurement: 1) differences between ACS patients; ${ }^{13-16} 2$ ) in association with ACS outcomes; $;^{17-20}$ 3 ) in relation to inflammation in ACS patients. ${ }^{21}$

The studies within each group are presented chronologically by publication date. All three HRV measurement categories are represented. Depression was diagnosed using criteria from the Diagnostic and Statistical Manual of Mental Disorders, 4th Edition (DSM-IV). The Hamilton Depression Scale (Ham-D), Beck Depression Inventory (BDI), and/or patient interviews determined severity. The study summaries are presented in Table 2 .

\section{Differences in HRV and depression between ACS patients}

Guinjoan et al prospectively investigated the connection between depression and abnormal cardiac autonomic activity in 56 patients who had had a recent ACS episode. ${ }^{13}$

Older ACS patients admitted to the CCU within the previous 3 days were recruited. Participants were interviewed and given depression scores based upon the Ham-D and criteria from the DSM-IV. The 56 patients were categorized into two groups: major depression or no depression. For the HRV analysis, morning ECG recordings of $\sim 5-10$ minutes 
(550 beats) were obtained using a device that detected $\mathrm{R}$-waves and analyzed the signals.

HRV time domain variables that reflected short-term beatto-beat variability ${ }^{24}$ were significantly different between the depressed and nondepressed groups, as were the percentage of normal RR intervals that were $>50$ milliseconds different from previous normal RR intervals ( $\mathrm{pNN} 50$ ) and the rootmean-square of successive RR differences (rMSNN). ${ }^{13}$

In the frequency domain, HF power was significantly lower in depressed patients and remained so in a multiple regression with clinical variables $(P<0.005)$. Total power, $\mathrm{LF}$, and the $\mathrm{LF} / \mathrm{HF}$ ratio were not significantly different between groups (note that this variable needs to be measured over 24 hours, and may not be accurate in a short recording, as outlined in Table 1). The authors concluded that depression was related to decreased vagal cardiac activity in older ACS patients. They noted that the sympathetic component could still be a factor but was dampened because most participants received betablocking medication.

Research by Vigo et $\mathrm{al}^{14}$ used the same database and data collection methods as did Guinjoan et al. ${ }^{13}$ Two nonlinear dynamics measures and one linear measure were compared between groups of depressed and nondepressed patients. The two nonlinear variables measured were: 1) approximate entropy (ApEn), a measure of randomness or disorder within a system, ${ }^{40}$ and 2 ) the short-term fractalscaling exponent, computed using detrended fluctuation analysis (DFA), a method that takes into account changing conditions over time and is useful in the analysis of physiologic signals ( $\alpha 1$ or DFA 1$){ }^{43,44}$ The linear measure was derived from a scatter plot of RR intervals, reflecting instantaneous beat-to-beat variation (SD1) (see Table 1 for definitions). ${ }^{47}$

SD1 and ApEn were lower and DFA1 was higher in depressed compared with nondepressed patients. The authors concluded that higher depression severity was related to reduced instantaneous beat-to-beat variability (SD1), increased randomness (ApEn), and increased fractal correlation properties (DFA1).

Catipović-Veselica et al performed a prospective study to examine the prevalence of minor and major depression in relation to HRV and clinical characteristics, in 297 hospitalized ACS patients. ${ }^{15}$ The patients underwent 24-hour ECG monitoring for HRV measurement; time domain HRV was computed. Interviews assessed the presence of major or minor depression, using DSM-IV criteria, prior to discharge 14-18 days after admission.
The time domain variables were significantly lower in depressed ACS patients compared with those who were not depressed. In addition, these investigators found that depression was more prevalent in women than men, corresponding to the findings of Carney et al. ${ }^{11}$ However, in contrast to the findings of Carney et al, Catipović-Veselica et al found that depression was more prevalent in older patients. ${ }^{15}$

Martens et al sought to examine the extent to which acute depressive and anxiety symptoms during hospitalization for acute MI could predict 24-hour time and frequency domain indices 2 months posthospitalization. ${ }^{16}$ In this study, 93 patients were screened for depression and anxiety during hospitalization, using the BDI and the State Trait Anxiety Inventory for self-report. In all, 82 patients were included in the 2-month follow-up visit, at which time they were assessed for major depressive disorder (MDD), using the Composite International Diagnostic Interview (CIDI); HRV was measured using 24-hour Holter recordings. Time and frequency domain indices were measured.

Nineteen patients were diagnosed with MDD using the CIDI; seven were diagnosed with anxiety disorder (three were diagnosed with both). Univariate analyses showed that the patients with depressive disorder had low SDNN and SDANN $(P<0.05)$; in similar analyses, patients with anxiety disorder had low rMSSD $(P<0.05)$ (see Table 1 for definitions). In multivariable analyses, adjusted for age, sex, previous cardiac history, and multivessel disease, anxiety disorder was found to be significantly associated with reduced rMSSD and HF power $(P<0.05)$, but MDD and HRV were not significantly associated. Neither depressive nor anxiety symptoms during hospitalization were significant predictors of HRV 2 months later.

\section{HRV and depression in association with ACS outcomes}

Research by de Guevara et al ${ }^{17}$ used the same database as did Guinjoan et $\mathrm{al}^{13}$ and Vigo et al. ${ }^{14}$ The investigators aimed to determine whether or not depressive symptoms and changes in autonomic activity, measured by HRV, are associated post-ACS, in older patients. The data were examined at two time points: in-hospital and at 6 months post-ACS. Of 48 patients recruited, 38 had complete follow-up information and were included in the analysis. Eight patients died. Those who died were significantly more likely to meet criteria for major depression at discharge than were patients who survived $(P=0.039)$. At 6 months, the survivors with depression scores that increased were more likely to have lower frequency domain HRV. The researchers concluded that cardiac autonomic function diminished with worsening 
of patient depressive symptoms at 6 months post-ACS episode.

Carney et al hypothesized that HRV mediates the effect of depression and designed a study to identify this effect in post-MI patients. ${ }^{18}$ The investigators proposed that the effect of HRV would be to mediate the pathway between depression and time to death, in ACS patients. The study was a secondary analysis of the Enhancing Recovery in Coronary Heart Disease (ENRICHD), a multicenter clinical trial, and was built upon the authors' previous work in which autonomic dysfunction was proposed as a potential physiological mechanism linking depression with increased mortality after MI. ${ }^{11,18}$

Consenting patients were screened 4 weeks after ACS hospitalization, using the BDI. Cognitive impairment or severe illness constituted exclusion criteria. Patients were considered depressed if they met the criteria for major or minor depression per the DSM-IV. HRV was measured with 24-hour Holter recorders. Based upon the authors' previous research, VLF was chosen for the HRV reference measure, and a risk score was derived from factors related to all-cause mortality, such as age, diabetes, and left ventricular ejection fraction. ${ }^{17}$ Follow-up occurred at 6 and 30 months. Allcause mortality was the primary end point; in all, 47 out of 678 patients died.

VLF was significantly lower in depressed compared with nondepressed patients in univariate analysis. The relationship stayed significant after adjustment with the risk score. In analysis of proportional hazard across time, inclusion of VLF was found to alter the risk of mortality related to depression. The proportion of the hazard of depression related to mortality risk that could be attributed to VLF was $27 \%$. The researchers concluded that low HRV is a partial mediator of the effect of depression in MI patient survival.

Drago et al sought to determine whether depressed ACS patients demonstrate cardiac autonomic dysfunction, as measured by HRV, and whether autonomic dysfunction had a mediating effect on depression in patients' 5 -year prognosis. ${ }^{19}$ The primary end points were recurrence of MI, need for revascularization, death, or a composite adverse event including any of the three. The investigators consecutively enrolled 100 acute MI patients at an Italian medical center. Psychiatric interviews and the BDI screened for depression 7-14 days after admission. DSM-IV diagnoses identified patients with MDD or mild-to-moderate depression. HRV was measured using 24-hour Holter monitors prior to discharge. Only SDNN was computed.

SDNN was significantly lower at baseline in patients with MDD $(P<0.01)$ or mild-to-moderate depression $(P=0.01)$ compared with those who were not depressed. Over 5 years,
30 adverse events occurred, including six deaths. In univariate analyses, SDNN was associated with adverse events, including death within five years. However, when clinical characteristics of the patients were included in a multivariate analysis, SDNN was no longer significantly associated with death or other adverse events. Depression was a significant contributor to all adverse outcomes, with age and sex controlled $(P=0.04)$. The authors concluded that depressed post-MI patients exhibited cardiac autonomic dysfunction, reflected by decreased SDNN, but this did not appear to be a mediator of depression in relation to 5-year outcomes.

Glassman et al studied the influence of sertraline (Zoloft; Pfizer, Inc., New York, NY, USA), a selective serotonin reuptake inhibitor, and mood improvement on HRV in ACS patients, using data from the Sertraline Antidepressant Heart Attack Randomized Trial (SADHART), an international, multicenter clinical trial. ${ }^{20}$ The Ham-D was used to measure depression severity; patients diagnosed with MDD were recruited for continuing participation and then were randomly selected to receive either sertraline or a placebo. HRV was assessed 3 weeks after hospitalization (baseline) and at 16 weeks, using 24-hour ECG recordings. Frequency domain measures, HF, LF, VLF, and ULF, were obtained. Mood change was assessed with the Clinical Global Impressions score, a scale that measures disease severity and improvement attributed to treatment response. ${ }^{48}$ Paired analyses of ECG recordings were performed for 258 patients.

Contrary to previous research showing the recovery of HRV indices in the months following $\mathrm{MI}^{49}$ neither group showed an improvement in HRV after 16 weeks. ULF was significantly lower in the placebo group $(P<0.05)$, and LF was significantly lower in the sertraline group $(P<0.05)$. However, when the two groups were compared with each other, ULF was significantly higher in the sertraline group because ULF was exceptionally low in the placebo group. Therefore, higher HRV was associated with the administration of sertraline and better mood. In addition, comparison of patient mood, independent of treatment, showed that mood improvement was closely associated with higher LF power, but this did not reach statistical significance. Low LF, in patients whose mood did not improve, was the primary driver in this association. The investigators concluded that depression impairs HRV recovery after MI.

\section{HRV and depression in relation to inflammation in ACS patients}

Frasure-Smith et al investigated connections between HRV, inflammation, and depression. Resting measures of three HRV indices (SDNN, HF, and LF, measured in 20-minute ECG 
recordings) and two inflammatory biomarkers (interleukin [IL]-6 and C-reactive protein) were examined in relation to the level of depressive symptoms in 682 post-ACS patients. ${ }^{21}$ The data were originally collected for the Epidemiological Study of Acute Coronary Syndromes and Pathophysiology of Emotions (ESCAPE).${ }^{50}$ Depressive symptoms were measured using the $\mathrm{BDI}$, and patients were assessed for MDD in interviews. HRV indices and inflammatory markers were obtained once during a follow-up visit 2 months after hospitalization.

The patients were categorized as depressed or nondepressed; a total of 47 met criteria for MDD. Analysis of the HRV and depressive symptoms did not reveal significant associations. However, SDNN, LF, and HF were significantly correlated with IL-6 in univariate analysis and remained significant when adjusted with clinical factors (such as age, sex, and previous MI). All three HRV variables were significantly related to C-reactive protein in univariate analysis; SDNN remained significant in the multivariate model $(P=0.044)$. The correlations between HRV and the inflammatory markers, especially $\mathrm{C}$-reactive protein, became stronger in association with greater depression. In the patients with higher depression scores, HRV accounted for $4 \%-5 \%$ of the variance in $\mathrm{C}$-reactive protein in the multivariate analysis. The authors concluded that HRV and inflammatory markers are elevated in association with post-MI patient depression.

\section{Discussion}

While several study results point toward the possibility of a relationship between HRV and depression in ACS patients, differences in design produced a range.

The findings from the first group of studies suggest a relationship between HRV and depression during an ACS episode. However, operational definitions and measurement methods varied, so drawing a definitive conclusion about significant relationships is thorny. While DSM-IV criteria identified depression in all the studies, the defining variable ranged from mild-to-moderate ${ }^{13,14}$ to MDD. ${ }^{15}$ Regarding HRV, Guinjoan et al and Vigo et $\mathrm{al}^{14}$ used 5-10 minute supine recordings; ${ }^{13}$ Catipović-Veselica et al and Martens et al examined 24-hour ambulatory recordings. ${ }^{15,16}$

A closer look at the findings of Vigo et al may provide insight into nonlinear dynamics. The researchers' results contrast with those of Kop et al, ${ }^{51}$ who used 24-hour ECG recordings to determine that DFA1 tended to be lower in older depressed adults; DFA1 $<1.0$ was associated with a higher risk of CV mortality over 15 years. Mäkikallio et al examined the relationship between nonlinear dynamic indices and sudden cardiac death. ${ }^{44}$ These investigators found that low DFA1 predicted cardiac death in patients $>65$ years of age. The risk of dying was 2.5 times greater in patients with DFA1 $<1.0$ compared with those with higher values. ${ }^{3}$ In addition, Mäkikallio et al found no significant difference in ApEn between patients with poor outcomes and those who were event-free. Stein et al also examined nonlinear measures of HRV. $^{45-47}$ Stein et al found that low DFA1 was associated with greater mortality risk in community-dwellling adults $\geq 65$ years. $^{47}$ The disparities between the findings of Vigo et al and those of other researchers' raises questions. Again differences in design and methods may explain the seemingly opposite results. Mäkikallio et al and Stein et al used 24-hour as opposed to 5- to 10-minute recordings, to examine the relationship between $\geq 10$-year outcomes and HRV in different populations. However, examination of clinical depression was not the primary focus in either Mäkikallio et al's ${ }^{44}$ or Stein et al's ${ }^{47}$ studies.

Tulppo et al showed that in healthy subjects, DFA1 could be altered in opposite directions, depending upon the type of exposure - cold face immersion (the measure decreased) or cold hand immersion (the measure increased). The researchers concluded that DFA1 captures a dynamic interplay between parasympathetic and sympathetic activity. ${ }^{43}$

The value of the work of Vigo et al may be in bringing attention to HRV nonlinear dynamics. According to Seely and Macklem, ApEn, the entropy variable, reflects the Second Law of Thermodynamics, namely that entropy or randomness increases in a system over time. ${ }^{40}$ High ApEn is associated with increased randomness, and low ApEn, as found in the study of Vigo et al, may reflect reduced randomness, ${ }^{20}$ which could translate to less movement or even gridlock, mirroring a clogged system or a clotted vessel.

While the studies by Martens et al and Catipović-Veselica et al appear to come to different conclusions about the association of HRV and depression, the univariate findings were similar. Both researchers found significant associations between time domain HRV variables and the diagnosis of depression measured within the same time frame. ${ }^{15,16}$ Martens et al went a step further by using a multivariate approach to assess the relationship; however, the lack of significant results could have been due to the small sample, as the authors noted.

In the second group of studies, de Guevara et al found evidence to indicate that depression and HRV may vary in association with each other post-ACS, ${ }^{17}$ potentially providing background for Carney et $\mathrm{al}^{18}$ and Drago et $\mathrm{al}^{19}$ to examine the mediating role of HRV on depression in mortality outcomes. Exactly how depression contributes to post-MI mortality remains unclear. Altered cardiac autonomic function, possibly due to the activation of sympathetic fibers and vagal 
withdrawal, may be one explanation. However, Carney et al and Drago et al came to different conclusions about the role of HRV in mediating the effect of depression on mortality. While Carney et al estimated that HRV accounts for approximately $25 \%$ of the effect of depression on mortality, Drago et al found that a low SDNN was associated with higher incidence of adverse events, including mortality, but was not a significant contributor in mediating the effect of depression on mortality. ${ }^{19}$ The differences may partially be attributed to methodology. Glassman et al found that decline in cardiac autonomic functioning post-ACS might be tempered by an appropriate mood-enhancing intervention. ${ }^{20}$ The underpinning for this finding is unclear, but the presence of the association may have promising implications for future treatments.

In the last study category, Frasure-Smith et al suggested the possibility of a mediating effect between HRV and inflammation in depressed ACS patients. ${ }^{21}$ The investigators built upon their previous work examining post-MI depression, ${ }^{7}$ and their findings were consistent with other studies suggesting a relationship between higher levels of inflammatory markers and reduced vagal activity, reflected by low HRV measurements. $^{51-53}$

While the extent of each patient's hazard may vary, an ACS diagnosis increases the risk of future adverse events. ${ }^{30,31}$ Considering the study findings in this review as a whole, there is a possibility that depression and disrupted HRV may heighten this risk, while interventions leading to improved mood and healthy HRV may lower the risk and improve long-term outcomes.

Figure 1 represents possible relationships, suggesting that:

- Assessment for depression and/or disrupted HRV, among the myriad of social, psychological, and physiological risks, may aid the identification of higher risk ACS patients.

- Inflammation, represented by inflammatory markers, may play a role in a potential relationship between HRV and depressive symptoms.

- Interventions leading to improved mood and/or more normal HRV might offer additional guidance to help move patients toward better coronary outcomes.

- The underlying mechanisms (including inflammation) by which depression and disrupted HRV potentially impact acute coronary syndrome outcomes remain an open question.

The variety of HRV measurement methods makes it impossible to directly compare the findings in these different studies. There is need for a common language, meaningful to bioengineers and clinicians alike, to help advance the validity and reliability of measurements. For example, Vigo et al described an association between depression and a "decreased complexity of the interbeat time series," 14 but the most meaningful point for advanced practice nurses and other practitioners is that the measurement may reflect poor cardiac autonomic integration. Furthermore, the precise physiology that a specific HRV parameter represents cannot be determined, and no single HRV measure can be considered ideal. Whether the different HRV variables refer to parasympathetic or sympathetic function, both, or other underlying physiology (for example, neuroendocrine changes) remains obscure. ${ }^{2}$

While investigators have sought to establish a "gold standard" for HRV, there remains inconsistency regarding the best approach. Kotecha et al used 5-minute HRV as a noninvasive marker to identify patients with obstructive coronary artery disease. These researchers measured HRV in the frequency domain and concluded that the 5-minute HRV test was clinically useful as a risk stratification tool. ${ }^{54}$ Recently, the recording time period of five minutes was used as a "gold standard" in studies to determine the accuracy of HRV derived from photoplethysmography. ${ }^{55,56}$

Additional research indicates that measurement of HRV in association with clinical depression may be useful in other populations, lending support for measurement in ACS. For example, patients with decompensated heart failure have been another focus of interest. In a study by Guinjoan et al, HRV was measured in heart failure patients who had had an MI, using short-term (5-minute) recordings. Frequency domain variables were successfully measured, and an association between abnormal HRV and depressive symptoms was found. ${ }^{57}$

Nonetheless, since the landmark study linking HRV with outcomes of myocardial infarction by Kleiger et al in 1987, ${ }^{3}$ research using 24-hour Holter recordings to obtain ECG markers of autonomic function has continued to be prevalent. ${ }^{58}$ Twenty-four-hour recordings, in which HRV can be averaged over 5-minute increments, have the potential to allow risk assessment with both short-term and long-term measurements. In addition, one study of MI patients suggested the ability to continuously monitor patient ECG over 24 hours, starting from the time they arrive in the Emergency Department, may be a reasonable approach for assessing autonomic function. ${ }^{59}$

The variety of methods employed for measuring depression presents another challenge, by limiting systematic comparability of the studies in this review. While all used DSM-IV criteria and interviews, four studies added the BDI, ${ }^{16,18,19,21}$ and four used the Ham-D. ${ }^{13,14,17,20}$ One study used the CIDI, ${ }^{16}$ and another reported assessment for depression 
in interviews only. ${ }^{15}$ The studies also varied in their definition of depression. Like HRV, results for depression can vary widely depending upon methodology. ${ }^{9}$ Across studies, sample sizes ranged widely, from 3,816 to 68,220 participants. Five studies analyzed data for $\leq 100$ ACS patients, ${ }^{13,14,16-18}$ possibly resulting in low statistical power and, leaving true differences undetected.

Could HRV assessed during ACS hospitalization offer information in conjunction with depression screening to assist in assessing risk after an episode of ACS?

The evidence presented here is modest and in some cases, conflicting. Besides inflammation, ${ }^{21,51-53}$ additional factors associated with HRV or clinical depression need to be considered as possible confounders, such as age, ${ }^{11,15,55,56}$ sex, ${ }^{11,15.60,61}$ genetics, ${ }^{62}$ and comorbidities. ${ }^{11}$ Another attenuating factor is the administration of medications, such as beta blockers, which are widely used in the treatment of ACS. Beta blockers can modulate the sympathetic response of patients in distress, ${ }^{63}$ and this factor needs to be considered in HRV interpretation. Other medications, such as benzodiazepines, also may play a role in the autonomic response and need to be considered in assessing HRV measurements. ${ }^{64}$ Therefore, drawing a definitive conclusion about the viability of HRV measurement in conjunction with clinical depression during an acute phase of ACS would be premature.

\section{Conclusion}

The underlying interaction between HRV and depression remains elusive, but the possibility of the two relating to each other in ACS with incremental impact on patients' prognosis has not been ruled out. Employing a multidimensional approach, the inclusion of early HRV measurement along with depression screening in risk assessment for ACS patients might be practical for discharge planning and worthy of further study.

\section{Limitations of this review}

This review focused on measurement as close as possible to the acute event, considering that obtaining measurements in the hospital during cardiac monitoring might be a practical approach. A small body of literature was reviewed, and only five studies performed measurements in the hospital, three of which measured HRV and depression in the same sample of patients. ${ }^{13,14,17}$ Evidence for measurement in patients with stable coronary artery disease, and support for best timing of HRV and depression assessment after ACS were not presented.

\section{Key points}

Results suggest:

- There may be a relationship between HRV and depression in ACS patients, possibly reflecting a disruption of autonomic cardiac function.

- Worse outcomes may be associated with depression and disrupted HRV in ACS patients.

- HRV may act as a mediator between depression and mortality in ACS patients.

- Improved HRV and enhanced mood may be related.

- Inflammation may play a role in these relationships.

\section{Future directions}

Improving outcomes for ACS patients is an essential component of tertiary prevention. Early identification of ACS patients with severely disrupted HRV and/or depression may help target those who need the most recovery assistance. The aims of future research might include the consolidation of similar studies, to calculate an effect size of HRV differences between clinically depressed and nondepressed ACS patients over time. Additional study of possible mediating factors, including the role of inflammation, might be productive. Further exploration of nonlinear dynamic variables may also be warranted. Prospective studies are needed. Finally, randomized clinical trials comparing the outcomes of patients receiving early HRV measurement and depression screening versus usual care could help establish whether or not adding HRV measurement to bedside monitoring, along with depression screening, could be clinically useful to enhance the assessment of long-term risk in ACS patients.

\section{Disclosure}

The authors report no conflicts of interest in this work.

\section{References}

1. Go AS, Mozaffarian D, Roger VL, et al; American Heart Association Statistics Committee and Stroke Statistics Subcommittee. Heart disease and stroke statistics - 2013 update: a report from the American Heart Association. Circulation. 2013;127(1):e6-e245.

2. Lahiri MK, Kannankeril PJ, Goldberger JJ. Assessment of autonomic function in cardiovascular disease: physiological basis and prognostic implications. J Am Coll Cardiol. 2008;51(18):1725-1733.

3. Kleiger RE, Miller JP, Bigger JT, Moss AJ. Decreased heart rate variability and its association with increased mortality after acute myocardial infarction. Am J Cardiol. 1987;59(4):256-262.

4. La Rovere MT, Bigger JT, Marcus FI, Mortara A, Schwartz PJ. Baroreflex sensitivity and heart-rate variability in prediction of total cardiac mortality after myocardial infarction. ATRAMI (Autonomic Tone and Reflexes After Myocardial Infarction) Investigators. Lancet. 1998;351(9101):478-484.

5. Lanza GA, Pedrotti P, Rebuzzi AG, Pasceri V, Quaranta G, Maseri A. Usefulness of the addition of heart rate variability to Holter monitoring in predicting in-hospital cardiac events in patients with unstable angina pectoris. Am J Cardiol. 1997;80(3):263-267. 
6. Buccelletti E, Gilardi E, Scaini E, et al. Heart rate variability and myocardial infarction: systematic literature review and metanalysis. Eur Rev Med Pharmacol Sci. 2009;13(4):299-307.

7. Frasure-Smith N, Lespérance F, Talajic M. Depression following myocardial infarction. Impact on 6-month survival. JAMA. 1993;270(15): $1819-1825$.

8. Barth J, Schumacher M, Herrmann-Lingen C. Depression as a risk factor for mortality in patients with coronary heart disease: a meta-analysis. Psychosom Med. 2004;66(6):802-813.

9. van Melle JP, de Jonge P, Spijkerman TA, et al. Prognostic association of depression following myocardial infarction with mortality and cardiovascular events: a meta-analysis. Psychosom Med. 2004;66(6): 814-822.

10. Thombs BD, Bass EB, Ford DE, et al. Prevalence of depression in survivors of acute myocardial infarction. J Gen Intern Med. 2006;21(1): $30-38$.

11. Carney RM, Blumenthal JA, Stein PK, et al. Depression, heart rate variability, and acute myocardial infarction. Circulation. 2001;104(17): 2024-2028.

12. Malik M. Assessment of cardiac autonomic regulation. Ann Noninvasive Electrocardiol. 2011;16(4):319-320.

13. Guinjoan SM, de Guevara MS, Correa C, et al. Cardiac parasympathetic dysfunction related to depression in older adults with acute coronary syndrome. J Psychosom Res. 2004;56(1):83-88.

14. Vigo DE, Nicola Siri L, Ladrón De Guevara MS, et al. Relation of depression to heart rate nonlinear dynamics in patients $>$ or $=60$ years of age with recent unstable angina pectoris or acute myocardial infarction. Am J Cardiol. 2004;93(6):756-760.

15. Catipović-Veselica K, Galić A, Jelić K, et al. Relation between major and minor depression and heart rate, heart-rate variability, and clinical characteristics of patients with acute coronary syndrome. Psychol Rep. 2007;100(3 Pt 2):1245-1254.

16. Martens EJ, Nyklícek I, Szabó BM, Kupper N. Depression and anxiety as predictors of heart rate variability after myocardial infarction. Psychol Med. 2008;38(3):375-383.

17. de Guevara MS, Schauffele SI, Nicola-Siri LC, et al. Worsening of depressive symptoms 6 months after an acute coronary event in older adults is associated with impairment of cardiac autonomic function. J Affect Disord. 2004;80(2-3):257-262.

18. Carney RM, Blumenthal JA, Freedland KE, et al. Low heart rate variability and the effect of depression on post-myocardial infarction mortality. Arch Intern Med. 2005;165(13):1486-1491.

19. Drago S, Bergerone S, Anselmino M, et al. Depression in patients with acute myocardial infarction: influence on autonomic nervous system and prognostic role. Results of a five-year follow-up study. Int $J$ Cardiol. 2007;115(1):46-51.

20. Glassman AH, Bigger JT, Gaffney M, Van Zyl LT. Heart rate variability in acute coronary syndrome patients with major depression: influence of sertraline and mood improvement. Arch Gen Psychiatry. 2007;64(9): $1025-1031$

21. Frasure-Smith N, Lespérance F, Irwin MR, Talajic M, Pollock BG. The relationships among heart rate variability, inflammatory markers and depression in coronary heart disease patients. Brain Behav Immun. 2009;23(8):1140-1147.

22. Pokorný J, Staněk V, Vrána M. Sudden cardiac death thirty years ago and at present. The role of autonomic disturbances in acute myocardial infarction revisited. Physiol Res. 2011;60(5):715-728.

23. Heart rate variability for risk stratification of life-threatening arrhythmias. American College of Cardiology Cardiovascular Technology Assessment Committee. J Am Coll Cardiol. 1993;22(3):948-950.

24. Heart rate variability: standards of measurement, physiological interpretation and clinical use. Task Force of the European Society of Cardiology and the North American Society of Pacing and Electrophysiology. Circulation. 1996;93(5):1043-1065.

25. Lombardi F, Stein PK. Origin of heart rate variability and turbulence: an appraisal of autonomic modulation of cardiovascular function. Front Physiol. 2011;2:95.
26. Carney RM, Freedland KE. Is there a high-risk subtype of depression in patients with coronary heart disease? Curr Psychiatry Rep. 2012;14(1):1-7.

27. Nicholson A, Kuper H, Hemingway H. Depression as an aetiologic and prognostic factor in coronary heart disease: a meta-analysis of 6362 events among 146538 participants in 54 observational studies. Eur Heart J. 2006;27(23):2763-2774.

28. Carney RM, Rich MW, teVelde A, Saini J, Clark K, Freedland KE. The relationship between heart rate, heart rate variability and depression in patients with coronary artery disease. J Psychosom Res. 1988;32(2):159-164.

29. Stein PK, Carney RM, Freedland KE, et al. Severe depression is associated with markedly reduced heart rate variability in patients with stable coronary heart disease. J Psychosom Res. 2000; 48(4-5):493-500.

30. Antman EM, Anbe DT, Armstrong PW, et al; American College of Cardiology/American Heart Association Task Force on Practice Guidelines (Writing Committee to Revise the 1999 Guidelines for the Management of Patients With Acute Myocardial Infarction). ACC/ AHA guidelines for the management of patients with ST-elevation myocardial infarction - executive summary: a report of the American College of Cardiology/American Heart Association Task Force on Practice Guidelines (Writing Committee to Revise the 1999 Guidelines for the Management of Patients With Acute Myocardial Infarction). Circulation. 2004;110(5):588-636.

31. Anderson JL, Adams CD, Antman EM, et al; 2011 Writing Group Members; ACCF/AHA Task Force Members. 2011 ACCF/AHA Focused Update Incorporated Into the ACC/AHA 2007 Guidelines for the Management of Patients With Unstable Angina/Non-ST-Elevation Myocardial Infarction: a report of the American College of Cardiology Foundation/American Heart Association Task Force on Practice Guidelines. Circulation. 2011;123(18):e426-e579.

32. Drew BJ, Califf RM, Funk M, et al; American Heart Association; Councils on Cardiovascular Nursing, Clinical Cardiology, and Cardiovascular Disease in the Young. Practice standards for electrocardiographic monitoring in hospital settings: an American Heart Association scientific statement from the Councils on Cardiovascular Nursing, Clinical Cardiology, and Cardiovascular Disease in the Young: endorsed by the International Society of Computerized Electrocardiology and the American Association of Critical-Care Nurses. Circulation. 2004;110(17):2721-2746.

33. Tsuji H, Venditti FJ, Manders ES, et al. Reduced heart rate variability and mortality risk in an elderly cohort. The Framingham Heart Study. Circulation. 1994;90(2):878-883.

34. Schneider RA; American Society for Clinical Investigation. Relationship of sinus arrhythmia to age and its prognostic significance in ischemic heart disease [abstract]. Clin Res. 1965;13:219.

35. Wolf MM, Varigos GA, Hunt D, Sloman JG. Sinus arrhythmia in acute myocardial infarction. Med J. 1978;2(2):52-53.

36. Kadish AH, Buxton AE, Kennedy HL, et al. ACC/AHA clinical competence statement on electrocardiography and ambulatory electrocardiography. A report of the ACC/AHA/ACP-ASIM Task Force on Clinical Competence (ACC/AHA Committee to Develop a Clinical Competence Statement on Electrocardiography and Ambulatory Electrocardiography). J Am Coll Cardiol. 2001;38(7): 2091-2100.

37. Akselrod S, Gordon D, Ubel FA, Shannon DC, Berger AC, Cohen RJ. Power spectrum analysis of heart rate fluctuation: a quantitative probe of beat-to-beat cardiovascular control. Science. 1981;213(4504):220-222.

38. Yasuma F, Hayano J. Respiratory sinus arrhythmia: why does the heartbeat synchronize with respiratory rhythm? Chest. 2004;125; 683-690.

39. Karasulu L, Dalar L, Sökücü S, Altın S. Heart rate variability analysis of single-channel electrocardiogram can help to differentiate high-risk patients with obstructive sleep apnea syndrome - a study on diagnostic accuracy. Anadolu Kardiyol Derg. 2012;12(4):331-338. 
40. Seely AJE, Macklem PT. Complex systems and the technology of variability analysis. Critical Care. 2004;8(6):R367-R384.

41. Babloyantz A, Destexhe A. Is the normal heart a periodic oscillator? Biol Cybern. 1988;58(3):203-211.

42. Bravi A, Longtin A, Seely AJ. Review and classification of variability analysis techniques with clinical applications. Biomed Eng Online. 2011;10:90.

43. Tulppo MP, Kiviniemi AM, Hautala AJ, et al. Physiological background of the loss of fractal heart rate dynamics. Circulation. 2005;112(3): 314-319.

44. Mäkikallio TH, Huikuri HV, Mäkikallio A, et al. Prediction of sudden cardiac death by fractal analysis of heart rate variability in elderly subjects. J Am Coll Cardiol. 2001;37(5):1395-1402.

45. Stein PK, Domitrovich PP, Hui N, Rautaharju P, Gottdiener J. Sometimes higher heart rate variability is not better heart rate variability: results of graphical and nonlinear analyses. $J$ Cardiovasc Electrophysiol. 2005;16(9):954-959.

46. Stein PK, Barzilay JI, Chaves PH, et al. Novel measures of heart rate variability predict cardiovascular mortality in older adults independent of traditional cardiovascular risk factors: the Cardiovascular Health Study (CHS). J Cardiovasc Electrophysiol. 2008;19(11):1169-1174.

47. Stein PK, Domitrovich PP, Huikuri HV, Kleiger RE; Cast Investigators. Traditional and nonlinear heart rate variability are each independently associated with mortality after myocardial infarction. $J$ Cardiovasc Electrophysiol. 2005;16(1):13-20.

48. Bandelow B, Baldwin DS, Dolberg OT, Andersen HF, Stein DJ. What is the threshold for symptomatic response and remission for major depressive disorder, panic disorder, social anxiety disorder, and generalized anxiety disorder? J Clin Psychiatry. 2006;67(9):1428-1434.

49. Bigger JT, Fleiss JL, Rolnitzky LM, Steinman RC, Schneider WJ. Time course of recovery of heart period variability after myocardial infarction. J Am Coll Cardiol. 1991;18(7):1643-1649.

50. Frasure-Smith N, Lespérance F, Irwin MR, Sauvé C, Lespérance J, Théroux P. Depression, C-reactive protein and two-year major adverse cardiac events in men after acute coronary syndromes. Biol Psychiatry. 2007;62(4):302-308.

51. Kop WJ, Stein PK, Tracy RP, Barzilay JI, Schulz R, Gottdiener JS. Autonomic nervous system dysfunction and inflammation contribute to the increased cardiovascular mortality risk associated with depression. Psychosom Med. 2010;72(7):626-635.

52. Carney RM, Freedland KE, Stein PK, et al. Heart rate variability and markers of inflammation and coagulation in depressed patients with coronary heart disease. J Psychosom Res. 2007;62(4):463-467.
53. Lanza GA, Sgueglia GA, Cianflone D, et al; SPAI (Stratificazione Prognostica dell'Angina Instabile) Investigators. Relation of heart rate variability to serum levels of C-reactive protein in patients with unstable angina pectoris. Am J Cardiol. 2006;97(12):1702-1706.

54. Kotecha D, New G, Flather MD, Eccleston D, Pepper J, Krum H. Five-minute heart rate variability can predict obstructive angiographic coronary disease. Heart. 2012;98(5):395-401.

55. Russoniello CV, Zhirnov YN, Pougatchev VI, Gribkov EN. Heart rate variability and biological age: implications for health and gaming. Cyberpsychol Behav Soc Netw. 2013;16(4):302-308.

56. Russoniello CV, Pougtachev V, Zhirnov E, Mahar MT. A measurement of electrocardiography and photoplethesmography in obese children. Appl Psychophysiol Biofeedback. 2010;35(3):257-259.

57. Guinjoan SM, Castro MN, Vigo DE, et al. Depressive symptoms are related to decreased low-frequency heart rate variability in older adults with decompensated heart failure. Neuropsychobiology. 2007; 55(3-4):219-224.

58. Huikuri HV, Stein PK. Heart rate variability in risk stratification of cardiac patients. Prog Cardiovasc Dis. 2013;56(2):153-159.

59. Harris PR, Stein PK, Fung GL, Drew BJ. Prognostic value of heart rate turbulence for risk assessment in patients with unstable angina and non-ST elevation myocardial infarction. Vasc Health Risk Manag. 2013;9:465-473.

60. Mallik S, Spertus JA, Reid KJ, et al; PREMIER Registry Investigators. Depressive symptoms after acute myocardial infarction: evidence for highest rates in younger women. Arch Intern Med. 2006;166(8): 876-883.

61. Zimmermann-Viehoff F, Orth-Gomer K, Wang HX, et al. Depressive symptoms and heart rate variability in younger women after an acute coronary event. Eur J Cardiovasc Prev Rehabil. 2010;17(5): 509-513.

62. Vaccarino V, Lampert R, Bremner JD, et al. Depressive symptoms and heart rate variability: evidence for a shared genetic substrate in a study of twins. Psychosom Med. 2008;70(6):628-636.

63. Jokinen V, Tapanainen JM, Seppänen T, Huikuri HV. Temporal changes and prognostic significance of measures of heart rate dynamics after acute myocardial infarction in the beta-blocking era. Am J Cardiol. 2003;92(8):907-912.

64. Unoki T, Grap MJ, Sessler CN, et al. Autonomic nervous system function and depth of sedation in adults receiving mechanical ventilation. $\mathrm{Am} \mathrm{J}$ Crit Care. 2009;18(1):42-50; quiz 51.
Neuropsychiatric Disease and Treatment

\section{Publish your work in this journal}

Neuropsychiatric Disease and Treatment is an international, peerreviewed journal of clinical therapeutics and pharmacology focusing on concise rapid reporting of clinical or pre-clinical studies on a range of neuropsychiatric and neurological disorders. This journal is indexed on PubMed Central, the 'PsycINFO' database and CAS.

\section{Dovepress}

The manuscript management system is completely online and includes a very quick and fair peer-review system, which is all easy to use. Visit http://www.dovepress.com/testimonials.php to read real quotes from published authors. 